\title{
Epidemiology and clinical characteristics of autoimmune hepatitis in the Netherlands
}

\begin{abstract}
NICOLE M.F. VAN GERVEN ${ }^{1}$, BART J. VERWER ${ }^{1}$, BIRGIT I. WITTE ${ }^{2}$, KAREL J. VAN ERPECUM ${ }^{3}$, HENK R. VAN BUUREN ${ }^{4}$, INGRID MAIJERS ${ }^{1}$, ARJAN P. VISSCHER ${ }^{1}$, EDWIN C. VERSCHUREN ${ }^{1}$, BART VAN HOEK ${ }^{5}$, MINNEKE J. COENRAAD ${ }^{5}$, ULRICH H.W. BEUERS ${ }^{6}$, ROBERT A. DE MAN ${ }^{4}$, JOOST P.H. DRENTH ${ }^{7}$, JANNIE W. DEN OUDEN ${ }^{8}$, ROBERT C. VERDONK ${ }^{9}$, GER H. KOEK $^{10}$, JOHANNES T. BROUWER ${ }^{11}$, MAUREEN M.J. GUICHELAAR ${ }^{12}$, JAN MAARTEN VROLIJK ${ }^{13}$, CHRIS J.J. MULDER ${ }^{1}$, CARIN M.J. VAN NIEUWKERK ${ }^{1}$ \& GERD BOUMA $^{1}$ ON BEHALF OF THE DUTCH AUTOIMMUNE HEPATITIS STUDY GROUP (SEE LIST OF CONTRIBUTORS AT THE END OF THE MANUSCRIPT)
\end{abstract}

\begin{abstract}
${ }^{1}$ Department of Gastroenterology and Hepatology, VU University Medical Center, Amsterdam, The Netherlands, ${ }^{2}$ Department of Epidemiology and Biostatistics, VU University Medical Center, Amsterdam, The Netherlands, ${ }^{3}$ Department of Gastroenterology and Hepatology, University Medical Center Utrecht, Utrecht, The Netherlands, ${ }^{4}$ Department of Gastroenterology and Hepatology, Erasmus University Medical Center, Rotterdam, The Netherlands, ${ }^{5}$ Department of Gastroenterology and Hepatology, Leiden University Medical Center, Leiden, The Netherlands, ${ }^{6}$ Department of Gastroenterology and Hepatology, Academic Medical Center, Amsterdam, The Netherlands, ${ }^{7}$ Department of Gastroenterology and Hepatology, Radboud University Nijmegen Medical Center, Nijmegen, The Netherlands, ${ }^{8}$ Department of Gastroenterology and Hepatology, Haga Hospital, The Hague, The Netherlands, ${ }^{9}$ Department of Gastroenterology and Hepatology, St. Antonius Hospital, Nieuwegein, The Netherlands, ${ }^{10}$ Department of Gastroenterology and Hepatology, University Medical Center Maastricht, Maastricht, The Netherlands, ${ }^{11}$ Department of Gastroenterology and Hepatology, Reinier de Graaf Medical Center, Delft, The Netherlands, ${ }^{12}$ Department of Gastroenterology and Hepatology, Medisch Spectrum Twente, Enschede, The Netherlands, and ${ }^{13}$ Department of Gastroenterology and Hepatology, Rijnstate Hospital, Arnhem, The Netherlands
\end{abstract}

\begin{abstract}
Background and aims. Epidemiological data on autoimmune hepatitis (AIH) are scarce. In this study, we determined the clinical and epidemiological characteristics of AIH patients in the Netherlands (16.7 million inhabitants). Methods. Clinical characteristics were collected from $1313 \mathrm{AIH}$ patients ( $78 \%$ females) from 31 centers, including all eight academic centers in the Netherlands. Additional data on ethnicity, family history and symptoms were obtained by the use of a questionnaire. Results. The prevalence of AIH was 18.3 (95\% confidential interval [CI]: 17.3-19.4) per 100,000 with an annual incidence of 1.1 (95\% CI: 0.5-2) in adults. An incidence peak was found in middle-aged women. At diagnosis, 56\% of patients had fibrosis and $12 \%$ cirrhosis in liver biopsy. Overall, $1 \%$ of patients developed HCC and 3\% of patients underwent liver transplantation. Overlap with primary biliary cirrhosis and primary sclerosing cholangitis was found in $9 \%$ and $6 \%$, respectively. The clinical course did not differ between Caucasian and non-Caucasian patients. Other autoimmune diseases were found in $26 \%$ of patients. Half of the patients reported persistent AIH-related symptoms despite treatment with a median treatment period of 8 years (range 1-44 years). Familial occurrence was reported in three cases. Conclusion. This is the largest epidemiological study of AIH in a geographically defined region and demonstrates that the prevalence of AIH in the Netherlands is uncommon. Although familial occurrence of $\mathrm{AIH}$ is extremely rare, our twin data may point towards a genetic predisposition. The high percentage of patients with cirrhosis or fibrosis at diagnosis urges the need of more awareness for AIH.
\end{abstract}

Key Words: autoimmune hepatitis, cirrhosis, diagnosis, diagnostic scoring systems, epidemiology, hepatocellular carcinoma, prevalence, transplantation

Correspondence: N.M.F. van Gerven, MD, Department of Gastroenterology and Hepatology, VU University Medical Center, De Boelelaan 1118, 1081 HV Amsterdam, The Netherlands. Tel: +31204440613. E-mail: n.vangerven@vumc.nl

(Received 11 May 2014; revised 8 fuly 2014; accepted 11 fuly 2014)

ISSN 0036-5521 print/ISSN 1502-7708 online @ 2014 Informa Healthcare DOI: $10.3109 / 00365521.2014 .946083$ 


\section{Introduction}

Autoimmune hepatitis (AIH) is a chronic inflammatory liver disorder of unknown etiology, which when left untreated can lead to hepatic failure and premature death [1]. Women are affected more frequently than men, and the disease can develop in all age groups. The clinical spectrum is wide, ranging from absent or mild symptoms to fulminant hepatic failure and may vary among ethnic groups [2]. AIH has features that resemble primary sclerosing cholangitis (PSC) and primary biliary cirrhosis (PBC) and overlap with these disorders are reported in $10-20 \%$ and $2-8 \%$ of cases, respectively $[3,4]$. Associated concomitant autoimmune diseases in AIH have been reported in up to $40 \%$ of patients and may mask the underlying liver disease [5].

There is no gold standard for the diagnosis of AIH, and diagnosis is based on the combination of clinical and laboratory findings and histological abnormalities after exclusion of viral and other causes of hepatitis [6]. Diagnostic scoring systems have been developed that help to make the diagnosis in the majority of patients and are particularly useful for defining homogenous patient groups in the context of studies [7]. A simplified scoring system with four variables was recently developed that enhances its applicability in daily practice [8].

There are few studies that have explored the prevalence of AIH. Older studies were often performed before the introduction of the diagnostic scoring system and have been biased to a certain extent by the fact that they do not rely on current standard criteria for patients' inclusion. Moreover, early studies did not exclude patients with chronic hepatitis C. Based on the available data, incidence of AIH is considered to be low, ranging from 0.08 in Japan [9] to 3 per 100,000 in the United Kingdom [10]. In the current study, we assessed the incidence and prevalence of AIH patients in the Netherlands 16.7 million inhabitants. In addition, we assessed the clinical spectrum and validity of diagnostic criteria in a large patient cohort of 1313 patients.

\section{Material and methods}

\section{Patient population}

Data for this retrospective study were retrieved from computerized or written medical records in 31 centers in the Netherlands, including 8 academic medical centers and 23 general district hospitals (Figure 1). Patients with AIH were identified based on information provided by co-investigators in the participating hospitals, by review of local AIH databases and by searching the database for international disease classification codes. The search was done in different

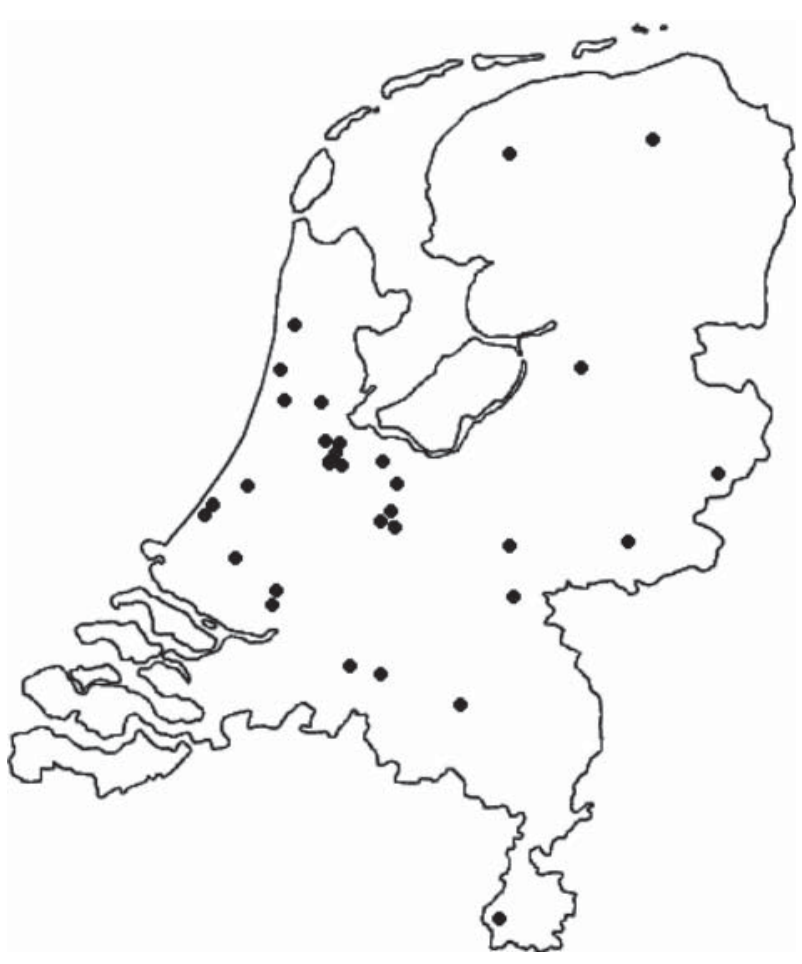

Figure 1. Figure-map of The Netherlands with locations of the hospitals included in this study.

local diagnostic registers in gastroenterology and internal medicine departments, but not in pediatrics. Adult patients who were diagnosed during infancy were likewise included. The medical records of AIH patients were traced back as long as possible. Patients included were diagnosed between 1967 and 2011.

The institutional review board of the VU University medical centre and the review boards of all participating centers approved the protocol. All participating patients gave written informed consent.

Diagnosis and application of diagnostic scoring systems

We included patients who had been diagnosed with AIH based on the combination of clinical, biochemical and histopathological criteria. In all patients, alcohol-induced and drug-related hepatitis had been excluded by clinical history. Viral hepatitis had been excluded by serological tests (hepatitis B and $\mathrm{C}$ ) in all cases. Diagnostic scores were determined according to both the International AIH Group (IAIHG) revised original criteria [7] and the simplified criteria [8]. In approximately half of the patients, not all characteristics were available to calculate the revised original criteria.

\section{Incidence and prevalence}

To accurately determine the incidence and prevalence, the Amsterdam geographic region was studied 
as catchment area. In this region, there are two academic hospitals and four regional hospitals. Many of the patients in the academic centers were referred from general district hospitals outside Amsterdam (50\%). Patients who were referred from outside the region were excluded from this analysis.

\section{Patient characteristics}

As all the data were collected from routine practice and diagnostic work-up, the available data could vary between participating hospitals. The following patient data were collected: gender, date of birth, date of diagnosis, characteristics of AIH (original and simplified criteria $[7,8]$ ) at diagnosis and duration and type of therapy. In the eight academic centers, it was documented if a liver transplantation was performed and when a patient developed a hepatocellular carcinoma (HCC). Laboratory data was including alanine-aminotransferase (ALT), immunoglobulin gamma $G$ (IgG), smooth mussel antibodies (SMA) and antinuclear antibodies (ANA) were collected.

Details on portal, interface, overall inflammatory activity and on fibrosis/cirrhosis were retrieved from pathology reports when liver biopsy at diagnosis had been performed.

With regard to concomitant autoimmune diseases, the diagnosis of inflammatory bowel disease (IBD) was based on clinical, endoscopic and histopathological features, diabetes mellitus on fasting blood glucose levels and thyroid disease by screening for TSH, T4 and when applicable thyroid antibodies. Diagnosis of overlap syndrome was made according to the diagnostic criteria of the involved diseases [3]. For PBC, these criteria composed of antimitochondrial antibody (AMA) titer higher than 1:80 and compatible histology. For PSC, these criteria generally were also based on histology and typical findings in radiological investigations (MRCP/ERCP). Human leukocyte antigen (HLA) typing was unavailable for all patients. Type of maintenance therapy was documented at the time the registers were searched.

Patients were asked to fill out a questionnaire assessing symptoms, family history and ethnicity. In monozygotic and dizygotic twins disease concordance was studied.

\section{Statistical analysis}

Summary statistics for continuous variables were expressed as median with range (minimum-maximum). Summary statistics for categorical variables were expressed as numbers (percentages). Quantitative variables were described as means with their standard deviations or as medians with their range if not normally distributed. Categorical variables were summarized with frequencies and percentages. Depending on the distribution, parametric and nonparametric tests, including t-test, Wilcoxon signed rank test and Sign exact test, were used to test for differences within and between groups. Cox regression univariate and multivariate analyses were used to test the relationship between patient characteristics and survival. The trend in incidence was measured by the Cochran-Armitage Test for Trend. Two-sided $p$-Values less than 0.05 were considered statistically significant. Statistical analyses were performed using Statistical Package for Social Sciences (SPSS).

\section{Results}

\section{Experimental design and cohort characteristics}

This retrospective analysis characterized the epidemiological characteristics of AIH in the Netherlands. A total of $1313 \mathrm{AIH}$ patients were identified; among these patients, $12 \%$ had been diagnosed under the age of 18 (Table I). Median follow-up time of this cohort was 10 years (range: $0-44$ years). There was a strong female preponderance; 1020 (78\%) were females. The median age at diagnosis in females was 48 years (range: 5-87 years) and in men 43 years (range: 687 years; $p>0.05$ ). An incidence peak in middle-aged women was found, with a smaller peak in adolescents (Figure 2). In men, no specific age-related incidence peak was observed.

The population of the Netherlands consists of 16.7 million inhabitants, of which $\sim 2$ million $(12 \%)$ are of non-Western descent (source: Statistics Netherlands; http://www/cbs.nl). This was reflected in the patient cohort. Among the 564 patients who reported their ethnic background in the questionnaire, 501 (89\%) were Caucasians, whereas $63(11 \%)$ of patients reported another background, mostly of African (34\%), Asian (16\%), South American (16\%) and European $(11 \%)$ descent. It has been reported that disease manifestations of AIH may vary among ethnic groups [11]. However, in this study cohort, no significant differences could be observed in the frequency of cirrhosis and fibrosis, ALT levels, antibodies, IAIHG disease scores according to the original and simplified criteria at presentation between Caucasian and non-Caucasian patients $(p>0.05$ for all parameters; data not shown).

\section{Incidence and prevalence}

Incidence and prevalence figures were determined in the Amsterdam area, covering a population of 
Table I. Patient characteristics in AIH and PBC/PSC overlap patients at diagnosis.

\begin{tabular}{lllll}
\hline Features & \multicolumn{1}{c}{ All patients } & \multicolumn{1}{c}{$\begin{array}{c}\text { Pure AIH } \\
(n=1110)\end{array}$} & $\begin{array}{c}\text { Overlap PSC/PBC } \\
(n=193)\end{array}$ \\
\hline Gender (female/male); $n$ & $1020 / 293$ & $865 / 255$ & $142 / 51$ & 1313 \\
Median age males; $y$ (range) & $43(6-87)$ & $43(8-87)$ & $40(6-82)$ & 293 \\
Median age females; $y$ (range) & $48(5-87)$ & $46(8-87)$ & $44(5-84)$ & 1020 \\
Median ALT; $I U / l$ (range) & $299(10-6667)$ & $368(20-6672)$ & $255(10-5523)$ & 1295 \\
Median IgG; $g / l$ (range) & $22(6-42)$ & $25(8-42)$ & $20(6-28)$ & 1206 \\
Median ALP; IU/l (range) & $149(16-7107)$ & $119(16-6204)$ & $263(111-7107)$ & 1270 \\
SMA pos; $n(\%)$ & $564(52 \%)$ & $530(58 \%)$ & $80(46 \%)$ & $1089(912 / 175)$ \\
ANA pos; $n$ (\%) & $610(53 \%)$ & $476(49 \%)$ & $88(49 \%)$ & $1152(972 / 180)$ \\
Cirrhosis/fibrosis (biopsy); $n(\%)$ & $128 / 580(12 \% / 56 \%)$ & $108 / 480(12 \% / 55 \%)$ & $20 / 100(12 \% / 58 \%)$ & $1041(869 / 172)$ \\
Decompensated liver cirrhosis; $n(\%)$ & $66(9 \%)$ & $59(9 \%)$ & $7(6 \%)$ & $730(657 / 115)$ \\
HCC; $n$ (\%) & $8(1 \%)$ & $7(1 \%)$ & $1(1 \%)$ & $730(657 / 115)$ \\
Liver transplantation; $n(\%)$ & $25(3 \%)$ & $19(3 \%)$ & $6(5 \%)$ & $772(657 / 115)$ \\
\hline
\end{tabular}

Abbreviations: $\mathrm{AIH}=$ Autoimmune hepatitis; $\mathrm{ALP}=$ Alkaline phosphatase; ALT = Alanine transaminase; ANA = Antinuclear antibodies; HCC = Hepatocellular carcinoma; IgG = Immunoglobulin G; PBC = Primary biliary cirrhosis; PSC = Primary sclerosing cholangitis SMA $=$ Smooth muscle antibodies.

799,000 individuals (data: Statistics Netherlands) and extrapolated to the rest of the country. A total of $146 \mathrm{AIH}$ patients were identified in the catchment area. According to these numbers the point prevalence in the Netherlands is 18.3 (95\% confidential interval [CI]: $17.3-19.4$ ) per 100,000.

The incidence was calculated over a time span of 10 years. Between 2000 and 2010, an average of 77 (range: 47-107) new AIH patients were diagnosed in the participating hospitals. An average of nine patients per year was diagnosed in the catchment area (Figure 3), resulting in an overall incidence in the Netherlands of 1.1 per 100,000 per year $(95 \%$ CI: $0.5-2)$. Remarkably, there was a significant rise in incidence over the past 10 years in both the catchment area $(p<0.01)$ and in the overall cohort $(p<0.01)$.

The incidence and prevalence of AIH was further confirmed using another approach. Based on the hospital size (inferred from the number of beds and referrals), it is estimated that the 23 large hospitals included in this study (out of a total of 99 hospitals in the Netherlands) serve approximately $45 \%$ of the population. Extrapolating the number of patients identified in the participating hospitals to the total population would result in a prevalence of 18.7 per 100,000 , very similar to the prevalence data tabulated from the catchment area.

\section{Scores according to the revised original [7] and simplified [8] AIH diagnostic criteria}

This analysis was performed in subgroups of patients with sufficient data to calculate the scores. Results of HLA typing were not available for our patients. The revised original criteria were assessed in 618 patients. The median post-treatment score at presentation in these patients was 19 points (range: 8-26 points). A total of 596 (97\%) of patients fulfilled the diagnostic criteria for $\mathrm{AIH}$; among these $442(72 \%)$ patients could be classified as definite AIH and $154(25 \%)$ as probable AIH. Among the 22 patients who did not fulfill the revised original criteria, $17(77 \%)$ had evidence for an overlap syndrome, in particular with PBC $(n=13)$. The fact that these patients did

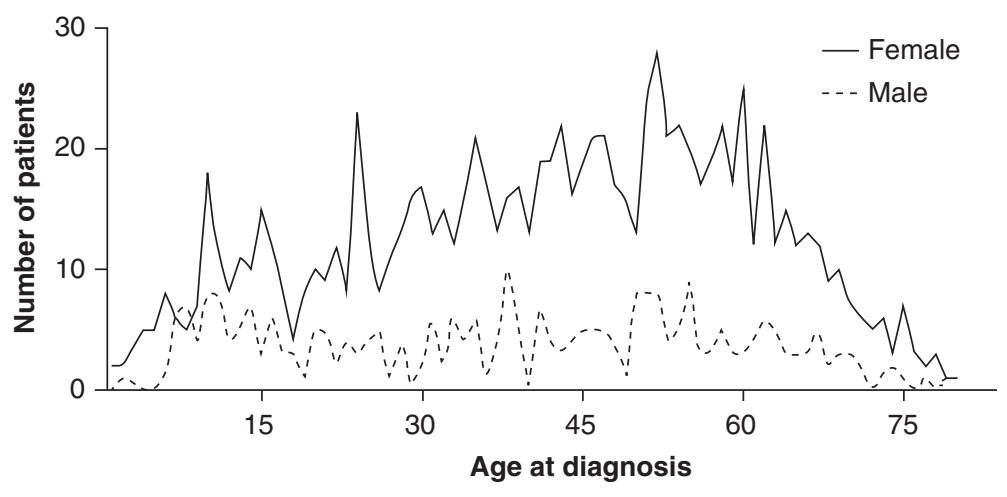

Figure 2. Age at diagnosis of AIH in males and females. 


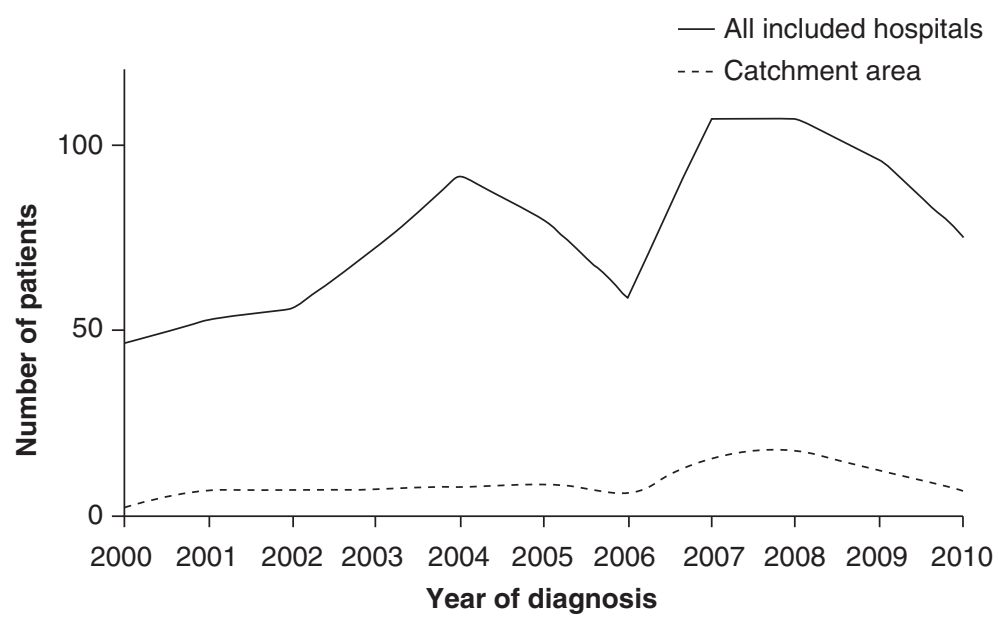

Figure 3. Incidence rates of AIH between 2000 and 2010.

not fulfill the diagnostic criteria for AIH can be attributed to the fact that in case of AMA positivity 4 points are subtracted from the total score.

A total of 749 patients could be classified according to the simplified criteria. The median score was 6.4 points (range: $2-8$ points). According to this classification, $171(23 \%)$ had probable AIH and 387 patients $(52 \%)$ definite $\mathrm{AIH}$, whereas 191 (25\%) patients did not fulfill the criteria for the diagnosis AIH (Table II). Among the patients who did not fulfill the diagnosis AIH according to the simplified criteria, 56 (29\%) had an overlap with either PBC $(n=32)$ or PSC $(n=24)$.

In 618 patients, parameters for both the revised original and the simplified criteria were available. Four hundred and seventy of these patients (76\%) fulfilled the criteria for either definite or probable AIH according to both scoring systems. A total of 110 patients (18\%) with AIH according to the revised original criteria ( 79 with probable and 31 with definite $\mathrm{AIH}$ ) did not fulfill the criteria for $\mathrm{AIH}$ according to the simplified criteria. Vice versa, six patients (five

Table II. Disease scores according to the revised original and simplified criteria.

\begin{tabular}{llcc}
\hline & & $\begin{array}{c}\text { All patients } \\
(\%)\end{array}$ & $\begin{array}{c}\text { Overlap with } \\
\text { PBC/PSC }\end{array}$ \\
\hline $\begin{array}{l}\text { Revised } \\
\text { original criteria } \\
n=618\end{array}$ & Definite AIH & $442(72 \%)$ & $41(9 \%)$ \\
& Probably AIH & $154(25 \%)$ & $34(22 \%)$ \\
$\begin{array}{l}\text { Simplified } \\
\text { criteria } \\
n=74\end{array}$ & No AIH & $22(3 \%)$ & $17(77 \%)$ \\
& Definite AIH & $387(52 \%)$ & $48(12 \%)$ \\
& Probable AIH & $171(23 \%)$ & $15(9 \%)$ \\
& No AIH & $191(25 \%)$ & $56(29 \%)$ \\
\hline
\end{tabular}

$\star$ Revised diagnostic scoring system of the international Autoimmune Hepatitis group (IAIHG) [7].

${ }^{\star \star}$ Simplified criteria of the IAIHG [8]. probable and one definite; $1 \%$ ) fulfilled the diagnosis for definite or probable AIH according to the simplified criteria, but not according to the revised original criteria (Table III).

\section{Laboratory values, antibodies and liver biopsy}

Laboratory values. In this cohort, ALT levels were above upper limit of normal (ULN) at diagnosis in $93 \%$ of patients, with a median ALT level of 299 IU/1 (range: 10-6672) (Table I). In 58\% of patients, ALP was not concomitantly elevated with ALP/ALT ratios $<1.5$ in $79 \%$ of all AIH patients.

Total serum IgG was above ULN in $83 \%$ of the patients with $60 \%$ of patients displaying levels $>1 \mathrm{~g} / 1$ above ULN. The median IgG level was $22 \mathrm{~g} / 1$ (range: 6-42).

In $36 \%$ of patients $(n=473)$, all three conditions $(\mathrm{ALT}>\mathrm{ULN}, \mathrm{ALP}<\mathrm{ULN}$ and $\mathrm{IgG}>\mathrm{ULN}$ ) were met at diagnosis.

Antibodies. ANA was measured in 1152 patients (88\%) and SMA in 1089 (83\%) at the time of diagnosis. Data on both antibodies could be retrieved from 1012 patients (77\%). In 53\% of patients, ANA was positive and in 52\% SMA positivity was found (Table I). Both antibodies were positive in $380(38 \%)$ of patients, whereas 861 patients $(85 \%)$ tested positive for at least one of these antibodies. liver kidney microsomal-1 antibodies (LKM-1) were measured in 78 patients. Four patients tested positive, three of them being under the age of 18 years.

Liver biopsy. In 1041 (79\%) patients, diagnosis was further substantiated by a liver biopsy. Piecemeal 
Table III. Comparison of the two scoring systems for the diagnosis AIH.

\begin{tabular}{llccrr}
\hline & & \multicolumn{3}{c}{ Patients with simplified criteria } \\
\cline { 3 - 5 } & & No AIH & Probable AIH & Definite AIH & Total \\
\hline Patients with revised original criteria & No AIH & 16 & 5 & 1 & $22(3 \%)$ \\
& Probable AIH & 79 & 33 & 42 & $154(25 \%)$ \\
& Definite AIH & 31 & 68 & 343 & $442(72 \%)$ \\
& Total & $126(20 \%)$ & $106(17 \%)$ & $386(63 \%)$ & 618 \\
\hline
\end{tabular}

${ }^{\star}$ Revised diagnostic scoring system of the international Autoimmune Hepatitis group (IAIHG) [7].

${ }^{\star \star}$ Simplified criteria of the IAIHG [8].

necrosis (interface hepatitis) was reported in 903 patients $(87 \%)$, plasma cells in 791 patients $(75 \%)$ and rosettes were described in 163 patients (16\%). At diagnosis, $580(56 \%)$ patients had fibrosis and $128(12 \%)$ patients had histological signs of cirrhosis (Table I). Rosettes and interface hepatitis were significantly more present in a biopsy with concomitant fibrosis or cirrhosis versus a biopsy without fibrosis or cirrhosis $(p<0.01$ and $p=0.01$, respectively).

In an effort to link the presence of fibrosis or cirrhosis to biochemical parameters, we observed that patients with a normal IgG had significantly less fibrosis and cirrhosis than patients with elevated IgG levels at the time of diagnosis $(p<0.05)$. No such association was found with ALT levels.

\section{Concurrent autoimmune disease}

Concomitant autoimmune diseases were observed in 335 patients (26\%). These included 121 (9\%) patients with thyroid disease, $63(5 \%)$ with IBD, 52 $(4 \%)$ with arthritis and $27(2 \%)$ with type I diabetes. Other reported autoimmune or immune-mediated diseases included celiac disease, autoimmune hemolytic anemia, sarcoidosis and multiple sclerosis. In this study, 39 (3\%) patients were diagnosed with more than one concomitant autoimmune disease. Significantly, more women than men $(27 \%$ vs. $21 \%$; $p=$ 0.02 ) had a concomitant other autoimmune disease.

\section{Overlap syndrome}

Concurrent features of PBC or PSC are frequently observed in AIH. In our AIH cohort, 113 of 1313 patients (9\%) were diagnosed with PBC and 80 patients $(6 \%)$ exhibited an overlap syndrome with PSC. Patient characteristics are summarized in Table I.

Biochemical and serological features of AIH were generally less pronounced in cases with overlap syndrome. Thus, mean ALT levels were lower in the group of patients with overlap syndrome (255 IU/1 [range: 10-5525] vs. 368 IU/1 [range:
20-6672]; $p<0.01)$. Similarly, the percentage of patients with ALT levels above ULN was lower in this patient category ( $87 \%$ vs $93 \%)$. In the overlap group, $72 \%$ had IgG levels above ULN with a mean IgG level of $20 \mathrm{~g} / 1$ (range: 6-28) versus $83 \%$ of patients without overlap with a mean level of $25 \mathrm{~g} / 1$ (range: $8-42)(p<0.01)$.

SMA was positive in $46 \%$ of overlap patients versus $58 \%$ in the remaining AIH patients $(p=0.04)$, whereas the frequency of ANA positivity did not differ between the two groups $(-50 \%)$.

\section{Self-reported disease onset and symptoms}

During the period, $38(3 \%)$ patients were diseased when the questionnaire was sent. A total of 564 (44\%) of the 1275 patients, including 472 females ( $84 \%$ ) and 92 males (16\%), returned the questionnaire. The selfreported information was collected a mean of 7 years after diagnosis (range: 1-25 years). A symptomatic disease onset was reported in $288(51 \%)$ of patients and included mainly jaundice (45\%) and fatigue $(51 \%)$ (Table IV). Forty-seven percent of patients

Table IV. Self-reported patient characteristics.

\begin{tabular}{|c|c|c|}
\hline Features & $n=564$ & $\begin{array}{l}\text { Percentage } \\
\quad(\%)\end{array}$ \\
\hline Gender (female/male) & $472 / 92$ & $84 / 16$ \\
\hline $\begin{array}{l}\text { Ethnic background } \\
\text { (Caucasian/other background) }\end{array}$ & $501 / 63$ & $89 / 11$ \\
\hline Symptomatic onset disease & 288 & 51 \\
\hline Fatigue & 130 & 45 \\
\hline Joint pain & 147 & 51 \\
\hline Abdominal pain & 26 & 9 \\
\hline Symptoms despite treatment: & 267 & 47 \\
\hline Fatigue & 165 & 62 \\
\hline Fatigue and abdominal pain & 30 & 11 \\
\hline Fatigue and joint pain & 22 & 8 \\
\hline Other & 50 & 19 \\
\hline $\begin{array}{l}\text { First-degree family member } \\
\text { with other autoimmune disease }\end{array}$ & 236 & 42 \\
\hline Direct family member with AIH & 2 & 0.3 \\
\hline \multicolumn{3}{|l|}{ Disease concordance in } \\
\hline Monozygotic twins $(n=1)$ & 1 & 100 \\
\hline Dizygotic twins $(n=5)$ & 0 & 0 \\
\hline
\end{tabular}


reported persistent AIH-related symptoms despite the instituted immunosuppressive treatment. The most common symptoms were fatigue, joint pain and abdominal pain (Table IV).

\section{Familial occurrence}

Data on familial occurrence and ethnicity were reported by 564 AIH patients. Among these, 236 patients $(42 \%)$ reported a first-degree family member with another autoimmune disease; thyroid disease (20\%), type I diabetes (14\%) and arthritis (9\%) being the most common. Two patients $(0.3 \%)$ had a relative with AIH. One patient had a mother diagnosed with $\mathrm{AIH}$ and the other a cousin. In addition, there was one monozygotic twinpair in this cohort, and both individuals had AIH. Among the five dizygotic twins, no disease concordance was found in any of the twin pairs (Table IV).

\section{Medication}

In 1284 patients, maintenance treatment was documented. A total of 627 patients (49\%) used combination therapy of prednisone and azathioprine, whereas 437 (34\%) used monotherapy (either prednisone or azathioprine). Around one-fifth of the patients $(17 \%)$ were not using maintenance therapy. In these 220 patients without maintenance therapy, 38 patients $(17 \%)$ did not satisfy the simplified or the revised original diagnostic criteria of the IAIHG. Patients using combination therapy had a higher mean diagnostic score at diagnosis (18.1 points according to the original criteria) when compared to patients on monotherapy (17.4 points) and patients without medication (14.2 points) $(p<0.01)$.

\section{Clinical course}

In the eight academic medical centers in the Netherlands (including all liver transplantation centers), the clinical course of 730 patients ( $76 \%$ female) was documented. The median follow-up time was 8 years (range 0-44 years). In these patients, 120 (16\%) developed portal hypertension, after a median of 17 months following diagnosis (range: 0 months38 years). Sixty-six patients (9\%) developed decompensated liver cirrhosis after a median of 16 months (range: 0 months-38 years). In this cohort, eight (1\%) patients were diagnosed with HCC, after a median of 6 years (range: 9 months-29 years) following diagnosis. Of the patients who developed a HCC, one patient had an overlap with PBC, none with PSC. Six of these patients had pre-existent cirrhosis.
Twenty-five (3\%) patients underwent liver transplantation after a median of 5 years (range: 8 months30years) following diagnosis. Five of these patients had an overlap with PSC, one with PBC. In addition, 14 patients $(2 \%)$ were screened for liver transplantation and are still on the waiting list.

\section{Discussion}

Epidemiological studies in AIH are scarce and the actual prevalence is currently unknown. Previous studies were hampered by the lack of uniform diagnostic criteria and were executed in either single centers or tertiary referral hospitals or consisted of mixed ethnic populations. In this large multi-centre survey, we aimed to overcome some of these limitations. In addition, we aimed to describe the clinical characteristics of AIH in a well-defined large cohort of patients. Hereto, patient records from not only all eight academic hospitals in the Netherlands were studied, as well as those from 23 regional nonacademic institutions population and comprises the largest epidemiological AIH study so far. Also, the catchment area for calculating incidence and prevalence included a combination of academic and non-academic hospitals and therefore presumably embodies a representative reflection of the overall AIH patient population. A mean annual incidence of 1.1 per 100.000 per year and a point prevalence in the Netherlands of 18.3 per 100.000 as calculated from the catchment area was found. It should be noted that the incidence data presented here might slightly underestimate true incidence figures since pediatric patients, who account for approximately $10 \%$ of patients, were not included in this survey. In addition, occasional patients may have been missed due to the fact that soluble liver antigen and LKM-1 antibodies are not routinely measured.

Although there is some variation, presumably due in part to the considerations mentioned above, the findings presented in this study are comparable to studies from other European countries, including Denmark, Norway, Sweden, the UK and Spain. Incidence rates in these populations vary between 0.8 and 3 and prevalence data between 12 and 17 [10,12-15]. A study from New Zealand reported an annual incidence of 2.0 cases of AIH per 100,000 and a somewhat higher prevalence of 24.5 cases per 100,000 [16]. A study in well-defined Alaskan native AIH patients reported a significantly higher prevalence of definite AIH of 35.9 cases per 100,000, with a particularly high prevalence in Southeast Indians. Unfortunately, no incidence data were presented in this study, and it should be noted that this study involved a limited number of 56 patients in a catchment area of 
100,312 individuals [17]. Finally in Japan, AIH appears to be considerably less frequent than in Western countries with an incidence estimated between 0.08 and 0.15 [18].

Of interest, a significant rise in incidence was seen in our cohort during the last decade and may indicate a true rise in incidence or, alternatively, increased awareness and diagnostic accuracy. These findings are in line with a previous observation from a single center study in Spain [14].

As expected, women were more affected than men with a sex ratio of 3.5:1 comparable to earlier studies [11]. The age of first disease manifestation varies greatly from early life until old age. In our cohort, the bimodal incidence pattern was only confirmed in women with a peak around the menopause [11]. Whether the incidence peak around menopause relates to hormonal changes as suggested in other autoimmune diseases warrants further research [19].

In our study population, $97 \%$ of patients satisfied the criteria for probable or definite AIH. The small group of patients who did not meet the criteria included a high number of PBC overlap patients. Although the original criteria have a high sensitivity, the application of this scoring system is cumbersome in daily clinical practice and therefore a simplified scoring system was developed. Three retrospective studies indicated that this simplified scoring system had a somewhat lower yet acceptable sensitivity when compared to the original revised criteria [20-22]. In this retrospective cohort, however, we observed that only $75 \%$ of patients with the clinical diagnosis $\mathrm{AIH}$ met the criteria for probable or definite AIH using this scoring system and in addition that almost one-fifth of patients who could be classified as definite AIH according to the revised original criteria did not fulfill the diagnosis $\mathrm{AIH}$ according to the simplified criteria. These findings indicate that at least in this large retrospective cohort the original revised criteria are superior to the simplified criteria. It should be noted that these findings are limited by the fact that comparison of these scoring systems was not the purpose of this study and that a significant number of patients could not be scored due to lack of data.

In all but its mildest form fibrosis is often present at diagnosis and with advanced disease bridging fibrosis and cirrhosis are often seen in AIH [11]. In our study cohort, more than half of the patients had fibrosis at diagnosis and in addition already $12 \%$ had cirrhosis. Similar or even higher cirrhosis rates have been observed in other studies, which indicate that disease has remained unrecognized for a significant period of time prior to diagnosis $[7,15]$. This study confirms earlier observations that the extent of histological damage is not related to the presence of symptoms [23] and thus urges the need for more awareness of the possibility of this disease even in asymptomatic patients.

Concurrent immune disorders are surprisingly common in AIH. One prospective study found that concurrent autoimmune disease was present in $28 \%$ of 122 patients [24]. The associated diseases may antedate the appearance of AIH or occur after the diagnosis has been established. The present study in a very large cohort of patients confirms these findings with thyroid disease being the most commonly associated disease. Similarly, the co-existence of PBC or PSC in this large cohort of patients is at the same order of magnitude as reported earlier in smaller cohorts (reviewed by Rust and Beuers that et al. [25]).

There are virtually no reliable data on familial occurrence in AIH. Yet the occurrence of AIH in members of the same family is an infrequent observation. One study explored familial occurrence in a cohort of 219 patients, and in this cohort, three cases were identified where both mother and daughter were affected [26]. There is an additional case report describing the occurrence of AIH in two sisters [27]. In the present study, we collected data from 564 patients by the use of a questionnaire. Cooccurrence of disease was identified in one motherdaughter pair and in addition in one third-degree relationship. Interestingly, the one monozygotic twinpair was concordant for disease, whereas no concordance was found in any of the five dizygotic twin pairs. Although these data are limited, all point, in addition to the established HLA association, towards a complex genetic predisposition, possibly with low penetrance [28]. This notion is further supported by the high frequency of other autoimmune disease, both within patients as well as in relatives of patients, as noticed in our patient cohort as well as in another study [29].

The development of HCC was previously regarded as a rare complication of $\mathrm{AIH}$. Three retrospective studies described the development of HCC in 1-4\% of AIH cases [30-32]. In a prospectively followed cohort, the rate HCC development was substantially higher with $6.2 \%$ of all patients. This number was as high as $9.3 \%$ in patients who had cirrhosis at presentation [33]. In the present study, these numbers are of the same magnitude as the earlier retrospective studies.

AIH represents the indication for liver transplantation in $4-6 \%$ of adults in both the US and Europe [34-37]. In our cohort, $3 \%$ of AIH patients underwent liver transplantation, whereas $2 \%$ were awaiting transplantation. This percentage is probably overestimated, while the data on transplantation were only collected from the academic centers and patients in need for transplantation are transferred to these centers. 
In conclusion, this study represents the largest nationwide study on the epidemiology of AIH so far. The findings presented in this study confirm that AIH is a relatively uncommon disease. The initial presentation of AIH varies widely, and diagnosis can be supported by the revised original criteria and, to a lesser extent, by the simplified criteria. During diagnosis, $12 \%$ of the patients already have cirrhosis and more than half of the patients fibrosis, which indicates a delay in diagnosis. No significant differences were observed in biochemistry, serologically and histology between Caucasian and non-Caucasian patients. Although familial occurrence is uncommon, the available data point towards the role of genetic factors in disease predisposition. Overall, $1 \%$ of AIH patients develop HCC and in 3\% of patients, liver a transplantation was performed. Prospective studies are warranted to substantiate the value of laboratory features, liver biopsy and to optimize the diagnostic criteria at diagnosis.

\section{Acknowledgment}

Collaborators: L.C. Baak (Onze Lieve Vrouwe Gasthuis, Amsterdam), M. Klemt-Kropp (Medisch Centrum Alkmaar, Alkmaar), J.J.M. van Meyel (Sint Lucas Andreas Ziekenhuis, Amsterdam), R.K. Linskens (St. Anna ziekenhuis, Geldrop), J.C. Kneppelhout (St. Anna ziekenhuis, Geldrop), J.Ph. Kuyvenhoven (Kennemer Gasthuis, Haarlem), E.J.M. van Geenen (Bronovo, den Haag,), M.J. Wagtmans (Rode Kruis ziekenhuis, Beverwijk), D.L. Cahen (ziekenhuis Amstelland, Amstelveen) F.H.J. Wolfhagen (Tweesteden ziekenhuis, Tilburg), P.J. Kingma (Tergooiziekenhuizen, Hilversum), J.M.L. de Vree (Medisch Centrum Leeuwarden, Leeuwarden), R.J.L.F. Loffeld (Zaans Medisch Centrum, Zaandam), J.M. Vrolijk (Rijnstate ziekenhuis, Arnhem), P.W. Friederich (Meander Medisch Centrum, Amersfoort), T.C.M. A. Schreuder (Slingeland ziekenhuis, Doetichem), A. W.M. van Milligen de Wit (Amphia ziekenhuis, Breda), M.A. Alleman (Isala, Zwolle), A. Bhalla (Hagaziekenhuis, Den haag), P.H.G.M. Stadhouders (St Antonius ziekenhuis, Nieuwegein), M.A.M.T. Verhagen (Diakonessenhuis, Utrecht).

Declaration of interest: The authors report no conflicts of interest. The authors alone are responsible for the content and writing of the paper.

\section{References}

[1] Czaja AJ. Current concepts in autoimmune hepatitis. Ann Hepatol 2005;4:6-24.
[2] Verma S, Torbenson M, Thuluvath PJ. The impact of ethnicity on the natural history of autoimmune hepatitis. Hepatology 2007;46:1828-35.

[3] Silveira MG, Lindor KD. Overlap syndromes with autoimmune hepatitis in chronic cholestatic liver diseases. Expert Rev Gastroenterol Hepatol 2007;1:329-40.

- [4] Teufel A, Weinmann A, Kahaly GJ, Centner C, Piendl A, Worns $\mathrm{M}$, et al. Concurrent autoimmune diseases in patients with autoimmune hepatitis. J Clin Gastroenterol 2010;44: 208-13.

[5] Gleeson D, Heneghan MA. British Society of Gastroenterology (BSG) guidelines for management of autoimmune hepatitis. Gut 2011;60:1611-29.

[6] Vergani D, Alvarez F, Bianchi FB, Cancado EL, Mackay IR, Manns MP, et al. Liver autoimmune serology: a consensus statement from the committee for autoimmune serology of the International Autoimmune Hepatitis Group. J Hepatol 2004;41:677-83.

[7] Alvarez F, Berg PA, Bianchi FB, Bianchi L, Burroughs AK, Cancado EL, et al. International Autoimmune Hepatitis Group Report: review of criteria for diagnosis of autoimmune hepatitis. J Hepatol 1999;31:929-38.

[8] Hennes EM, Zeniya M, Czaja AJ, Pares A, Dalekos GN, Krawitt EL, et al. Simplified criteria for the diagnosis of autoimmune hepatitis. Hepatology 2008;48:169-76.

- [9] Toda G, Watanabe F, Zeniya M. [Autoimmune hepatitis: an epidemiological study]. Nihon Naika Gakkai Zasshi 1999; 88:572-7.

[10] Whalley S, Puvanachandra P, Desai A, Kennedy H. Hepatology outpatient service provision in secondary care: a study of liver disease incidence and resource costs. Clin Med 2007; 7:119-24.

[11] Manns MP, Czaja AJ, Gorham JD, Krawitt EL, MieliVergani G, Vergani D, et al. Diagnosis and management of autoimmune hepatitis. Hepatology 2010;51:2193-213.

[12] Boberg KM, Aadland E, Jahnsen J, Raknerud N, Stiris M, Bell $\mathrm{H}$. Incidence and prevalence of primary biliary cirrhosis, primary sclerosing cholangitis, and autoimmune hepatitis in a Norwegian population. Scand J Gastroenterol 1998;33: 99-103.

[13] Gronbaek L, Vilstrup H, Jepsen P. Autoimmune hepatitis in Denmark: Incidence, prevalence, prognosis, and causes of death. A nationwide registry-based cohort study. J Hepatol 2014;60:612-17.

[14] Primo J, Merino C, Fernandez J, Moles JR, Llorca P, Hinojosa J. [Incidence and prevalence of autoimmune hepatitis in the area of the Hospital de Sagunto (Spain)]. Gastroenterol Hepatol 2004;27:239-43.

[15] Werner M, Prytz H, Ohlsson B, Almer S, Bjornsson E, Bergquist A, et al. Epidemiology and the initial presentation of autoimmune hepatitis in Sweden: a nationwide study. Scand J Gastroenterol 2008;43:1232-40.

[16] Ngu JH, Bechly K, Chapman BA, Burt MJ, Barclay ML, Gearry RB, et al. Population-based epidemiology study of autoimmune hepatitis: a disease of older women? J Gastroenterol Hepatol 2010;25:1681-6.

[17] Hurlburt KJ, McMahon BJ, Deubner H, Hsu-Trawinski B, Williams JL, Kowdley KV. Prevalence of autoimmune liver disease in Alaska Natives. Am J Gastroenterol 2002;97:2402-7.

[18] Toda G, Zeniya M, Watanabe F, Imawari M, Kiyosawa K, Nishioka M, et al. Present status of autoimmune hepatitis in Japan - correlating the characteristics with international criteria in an area with a high rate of HCV infection. Japanese National Study Group of Autoimmune Hepatitis. J Hepatol 1997;26:1207-12. 
[19] Sammaritano LR. Menopause in patients with autoimmune diseases. Autoimmun Rev 2012;11:A430-6.

[20] Czaja AJ. Performance parameters of the diagnostic scoring systems for autoimmune hepatitis. Hepatology 2008;48: 1540-8.

[21] Qiu D, Wang Q, Wang H, Xie Q, Zang G, Jiang H, et al. Validation of the simplified criteria for diagnosis of autoimmune hepatitis in Chinese patients. J Hepatol 2011;54: 340-7.

[22] Yeoman AD, Westbrook RH, Al-Chalabi T, Carey I, Heaton ND, Portmann BC, et al. Diagnostic value and utility of the simplified International Autoimmune Hepatitis Group (IAIHG) criteria in acute and chronic liver disease. Hepatology 2009;50:538-45.

[23] Kogan J, Safadi R, Ashur Y, Shouval D, Ilan Y. Prognosis of symptomatic versus asymptomatic autoimmune hepatitis: a study of 68 patients. J Clin Gastroenterol 2002;35:75-81.

[24] Czaja AJ, Carpenter HA, Santrach PJ, Moore SB. Genetic predispositions for immunological features in chronic liver diseases other than autoimmune hepatitis. J Hepatol 1996; 24:52-9.

[25] Rust C, Beuers U. Overlap syndromes among autoimmune liver diseases. World J Gastroenterol 2008;14:3368-73.

[26] Findor JA, Sorda JA, Daruich JR, Manero EF. Familial association in autoimmune liver disease. Medicina (B Aires) 2002;62:241-4.

[27] Ohira H, Shinzawa J, Suzuki T, Tojo J, Sato H, Nishimaki T, et al. Two sister cases of autoimmune hepatitis. Fukushima J Med Sci 1998;44:113-20.

[28] de Boer YS, van Gerven NM, Zwiers A, Verwer BJ, van Hoek B, van Erpecum KJ, et al. Genome-wide association study identifies variants with autoimmune hepatitis type-1. Gastroenterology 2014;147:443-52.
[29] Mieli-Vergani G, Heller S, Jara P, Vergani D, Chang MH, Fujisawa T, et al. Autoimmune hepatitis. J Pediatr Gastroenterol Nutr 2009;49:158-64.

[30] Montano-Loza AJ, Carpenter HA, Czaja AJ. Predictive factors for hepatocellular carcinoma in type 1 autoimmune hepatitis. Am J Gastroenterol 2008;103:1944-51.

[31] Teufel A, Weinmann A, Centner C, Piendl A, Lohse AW, Galle PR, et al. Hepatocellular carcinoma in patients with autoimmune hepatitis. World J Gastroenterol 2009; 15:578-82.

[32] Werner M, Almer S, Prytz H, Lindgren S, Wallerstedt S, Bjornsson E, et al. Hepatic and extrahepatic malignancies in autoimmune hepatitis. A long-term follow-up in 473 Swedish patients. J Hepatol 2009;50:388-93.

[33] Yeoman AD, Al-Chalabi T, Karani JB, Quaglia A, Devlin J, Mieli-Vergani G, et al. Evaluation of risk factors in the development of hepatocellular carcinoma in autoimmune hepatitis: Implications for follow-up and screening. Hepatology 2008;48:863-70.

[34] Cross TJ, Antoniades CG, Muiesan P, Al-Chalabi T, Aluvihare V, Agarwal K, et al. Liver transplantation in patients over 60 and 65 years: an evaluation of long-term outcomes and survival. Liver Transpl 2007;13:1382-8.

[35] Khalaf H, Mourad W, El-Sheikh Y, Abdo A, Helmy A, Medhat $Y$, et al. Liver transplantation for autoimmune hepatitis: a single-center experience. Transplant Proc 2007;39:1166-70.

[36] Sanchez-Urdazpal L, Czaja AJ, van Hoek B, Krom RA, Wiesner RH. Prognostic features and role of liver transplantation in severe corticosteroid-treated autoimmune chronic active hepatitis. Hepatology 1992;15:215-21.

[37] Seaberg EC, Belle SH, Beringer KC, Schivins JL, Detre KM. Liver transplantation in the United States from 1987-1998: updated results from the Pitt-UNOS Liver Transplant Registry. Clin Transpl 1998;17-37.

\section{Notice of correction}

The version of this article published online ahead of print on 15 Aug 2014 contained a number of minor typographical errors. These have been corrected for this version. A new reference has also been added in at number 28. Reference citations and the reference list have been updated accordingly. 